\title{
Stroke: Cutting It Off at the Pass
}

\author{
By Jack M. Gorman, MD
}

The mammalian brain is carefully protected by what is commonly referred to as the "blood-brain barrier." What this really represents is the special and complex relationship between the brain and its surrounding circulation. Like all organs of the body, the brain relies on oxygen and glucose delivered by arteries and capillaries, and on veins to carty away carbon dioxide. In fact, the metabolic rate of the brain is directly dependent on the rate of blood flow, giving rise to our ability to measure regional brain function with modern neuroimaging technologies.

Because the brain is the most complex and sensitive organ of the body, it cannot be exposed to all elements carried in circulating blood. Therefore, a dynamic but highly protective relationship between capillaries and astrocytes carefully filters what reaches the brain. Nimmerjahn and colleagues ${ }^{1}$ recently showed for the first time how another brain cell, the microglial cell, makes an immediate response to tiny tears in nearby capillaries by sending out filopodia-like processes that gobble up potentially neurotoxic substances leaking into the brain parenchyma and also seal the hole until coagulation can occur. While this response is mostly protective, it confounds some therapeutic attempts, as drugs that easily penetrate other organs are often unable to pass through the blood-brain barrier to their needed site of action. Oncologists know this well: chemotherapy for neoplasms in the central nervous system often must be administered intrathecally.

Disruptions of this delicate balance between the brain and its blood supply constitute the group of catastrophic events known as cerebrovascular accidents or stroke. The three main types of stroke (thrombotic, embolic, and hemorrhagic) have different features but collectively account for one of the leading causes of morbidity and mortality in the world. The brain has remarkable plasticity; ischemic damage to a part of the brain can often be compensated for by other areas. Nevertheless, strokes often leave their victims paralyzed, speechless, confused, or dead.

In the past, there was not much to do when the signs of stoke first emerged other than watchful waiting and basic life support. Patients suffering strokes were quickly admitted to the hospital for observation. The real work for those who survived was, and still very much is, the long and often tedious (for the patient) process of rehabilitation. Skilled physiatrists and physical therapists can realize dramatic improvement, but the work can take months and is often emotionally demanding for patients and their families.

Only recently have treatments that can soften the effects of an evolving stroke been developed. Invasive procedures to break up clots and unblock arteries leading to the brain, if done within hours of the first sign of a stroke, can result in far less subsequent damage. What was once a question of waiting has become one of how quickly a patient can be whisked to a hospital emergency room, evaluated, scanned, and brought to the intervention suite. Even so, most patients will require months of arduous rehabilitation therapy to regain anything similar to their original function.

With these dramatic improvements in treatment has come an ever-deepening appreciation of the risk factors for stroke. For the most part, the risk factors are similar to those for heart attack and, as we now know, for Alzheimer's disease. Cigarette smoking, diabetes, hypertension, obesity, hyperlipidemia, and lack of exercise all increase and individual's risk for stroke. Sadly, the physician who spends time eliciting these risk factors and undertakes the frustrating process of attempting to induce his or her patients to change their behavior to reduce or eliminate them will be poorly compensated. Better compensation goes to the physicians involved in heroic measures such as catheterizing a cerebral vessel and injecting tissue plasminogen activator to dissolve a thrombotic plaque or embolism. Our peculiar system of healthcare finance seems to prefer to wait for catastrophes to happen before spending money; one wonders what would happen if more money was spent on using the knowledge we have to increase primary prevention.

For all of the neurologists and psychiatrists reading this, remember that despite the fact that you cannot bill for sound advice, trying to talk your patients into losing weight; lowering their cholesterol; quitting smoking, taking their statins, antihypertensive medications, and aspirin; and getting exercise is one of the most noble enterprises a physician can undertake. CXS

\section{REFERENCE}

1. Nimmerjahn A, Kirchhoff F, Helmchen F. Resting microglial cells are highly dynamic surveillants of brain parenchyma in vivo. Stroke. 2005;308:1314-1318.

Dr. Gorman is the editor of CNS Spectrums and Esther and Joseph Klingenstein Professor of Psychiatry and chair of the Department of Psychiatry at Mount Sinai School of Medicine in New York City. 